\title{
El salto cuántico en el deporte mundial y su impacto social en Colombia
}

\section{The quantum jump in world sport and its social impact on Colombia}

\author{
Álvaro José Gracia-Díaz' (D) \\ 'Universidad de Ciencias Aplicadas y Ambientales - U.D.C.A, Bogotá, Colombia; agracia@udca.edu.co
}

Cómo citar: Gracia-Díaz, ÁJ. 2021. El salto cuántico en el deporte mundial y su impacto social en Colombia. Rev. Digit. Act. Fis. Deport. 7(1):e1794. http://doi.org/10.31910/rdafd.v7.n1.2021.1794

Artículo de acceso abierto publicado por Revista Digital: Actividad Física y Deporte, bajo una licencia Creative Commons CC BY-NC 4.0

Publicación oficial de la Universidad de Ciencias Aplicadas y Ambientales U.D.C.A, Institución de Educación Superior Acreditada de Alta Calidad por el Ministerio de Educación Nacional.

Recibido: Septiembre 17 de 2020 Aceptado: Octubre 27 de 2020 Editado por: Álvaro José Gracia Díaz

\section{RESUMEN}

Introducción: Las Nuevas Disciplinas Deportivas han nacido y se han extendido por todo el planeta tierra, a través de procesos de globalización que, al mismo tiempo, han promovido novedosas prácticas de ejercicio y de construcción corporal, caracterizados por su flexibilidad, creatividad, improvisación, recursividad e impacto social. Originados en la combinación de los deportes existentes y en la creación de actividades físicas nuevas, los nuevos deportes proponen enfatizar el disfrute antes que la competencia, para lograr mejores experiencias humanas. Objetivo general: La investigación, se propuso analizar la proyección social de las nuevas disciplinas deportivas en Colombia, a fin de dimensionar su impacto real. Metodología: Se adelantó un estudio exploratorio sobre las diferentes visiones existentes acerca del tema, por medio de una revisión documental de variadas fuentes nacionales e internacionales, cuyos productos se ofrecen en este artículo. Resultados: Lo más importante es que se ha logrado brindar al país nuevas perspectivas para abordar, promover y preparar las nuevas generaciones, en el disfrute de estas nuevas vivencias deportivas. Conclusiones: Con base en la reflexión profunda, se llegó a varias inferencias, conducentes a proponer cambios que pueden aportar los elementos necesarios para adelantar la actualización, tan necesaria en las facultades de cultura física y para fortalecer los procesos de investigación sobre los tópicos más pertinentes en este campo, de la vida social y cultural colombiana.

Palabras clave: Nuevas Disciplinas Deportivas; Cultura física; Sociedad; Pedagogía; Lúdica.

\section{ABSTRACT}

Introduction: The New Sports Disciplines have been born and spread throughout planet through globalization processes that, at the same time, have promoted novel practices of exercise and body construction characterized by their flexibility, creativity, improvisation, recursion and social impact. Originating in the combination of existing sports and the creation of new physical activities, new sports propose to emphasize enjoyment over competition to achieve better human experiences. General objective: The research set out to analyze the social projection of the new sports disciplines in Colombia in order to dimension their real impact. Methodology: An exploratory study on different existing visions on the subject was advanced through a documentary 
review of various national and international sources whose products are offered in this article. Results: The most important thing is that it has been possible to give the country new perspectives to address, promote and prepare the new generations in the enjoyment of these new sporting experiences. Conclusions: Based on deep reflection, several inferences were reached leading to propose changes that can provide the necessary elements to advance the update, so necessary in faculties of physical culture and to strengthen research processes on the most relevant topics in this field of Colombian social and cultural life.

Keywords: New Sports Disciplines; Physical culture; Society; Pedagogy; Playful.

\section{INTRODUCCIÓN}

El presente artículo de revisión presenta los resultados de la exploración documental acerca de las nuevas disciplinas deportivas surgidas en el mundo, a partir de la globalización. Igualmente, analiza el impacto social de los nuevos deportes en Colombia.

Cabe destacar la pertinencia del tema en una sociedad tan cambiante, ávida de explicaciones, sobre tantos fenómenos producidos por los sorprendentes y acelerados cambios científicos y tecnológicos, particularmente, en el mundo del deporte, que han permeado las formas y las costumbres sociales, alterando, incluso, hasta las maneras cotidianas de asumir la cultura física, a partir de las nuevas visiones surgidas de tales innovaciones.

Sin duda alguna, los resultados de esta investigación ofrecen a la población y más particularmente a las facultades de educación deportiva y demás instituciones dedicadas a la formación en cultura física y deporte, herramientas más sofisticadas y útiles, para el cumplimiento de su misión corporativa.

Finalmente, se hallarán las respuestas a muchas preguntas relacionadas con los principales acontecimientos deportivos de los últimos treinta años y, de ser así, es sentir la satisfacción del esfuerzo realizado y los resultados alcanzados.

\section{MATERIALES Y MÉTODOS}

Con base en la pregunta del estado de la proyección social en Colombia, en sus principales ciudades, acerca de las nuevas disciplinas deportivas surgidas con la globalización y con el objetivo de analizar su alcance, se realizó la revisión bibliográfica de textos y artículos científicos, relacionados con el tema, a nivel local, regional, nacional e internacional, para lo cual, se seleccionaron un total de 62 artículos funcionales, entre los 5.607 explorados y hallados en las diferentes bases de datos, completamente actualizadas, como Scopus, ProQuest (U.D.C.A), Google Académico, SportDiscus (U.D.C.A), ScienceDirect (U.D.C.A) y Scielo, además de libros y documentación disponibles sobre el tema en cuestión, en el mundo de hoy. Estos documentos, se dividen de la siguiente manera, Scopus (2), ProQuest (4), Google Académico (28), SportDiscus (10), ScienceDirect (5) y Scielo (13). La información allí contenida facilitó la tarea de la revisión.

De otro lado, y para hacer más productivo el ejercicio exploratorio, se aplicó una metodología basada en el enfoque cualitativo, de acuerdo con la visión de Hernández et al. (2014), por tratarse del procesamiento de múltiples y variados puntos de vista, acerca de las nuevas disciplinas deportivas, además de un diseño de revisión documental adecuado, para obtener los mejores resultados de una investigación basada en el manejo de múltiples fuentes.

\section{De la competencia al disfrute}

"Lo que legítima las nuevas formas de deportes, en particular, es la emoción corporal, el placer que debe ser experimentado aquí y ahora, la autoexpresión, lo divertido, la forma física y psicológica", Silvia Capretti.

Desde mitades del siglo XX e inicios del XXI, la práctica deportiva ha venido evolucionando, pasando de las perspectivas tradicionales a concepciones innovadoras, cercanas a una mayor población, hacia diversos medios, como el natural, el urbano, el extremo, la aventura. Algunos investigadores, como García (2013), hacen una clasificación en juegos y deportes colectivos, juegos y deportes de adversario, deportes individuales sobre ruedas, juegos de lanzamiento y juegos de cooperación. Las razones de esta actualización están ligadas con varios temas, entre ellos, el desarrollo cultural y socioeconómico, dado que se encuentra la necesidad del bienestar, la calidad de vida de la sociedad en los países con mayor desarrollo económico, lo que impulsa el consumo de servicios y la utilización del tiempo libre, induciendo 
a la creación, práctica y consumo de nuevos deportes (Fuster et al. 2008).

Otro motivo es el prototipo territorial que selecciona la población para la realización de actividades económicas y del trabajo cotidiano, obteniendo una alta concentración de personas en un medio urbano, generando un estilo de vida apresurado y monótono, lo que empuja, en primer lugar, a la actividad de deportes en los medios de aventura, naturales, extremos y novedosos, que logran sacar a las personas de ese automatismo, del día a día, en las ciudades. Y, en segundo lugar, el aprovechamiento del desarrollo y las estructuras urbanas para la práctica de actividades físico-deportivas, de talla competitiva y hasta federativa, reconocido como una expresión recreativa, improvisada, adaptada al medio urbano (García \& Fernández-Truan, 2018; Radojević et al. 2019).

Además, una de las razones más importantes de su aceptación es la comprensión por parte de organismos internacionales, de que el deporte puede contribuir a superar las dificultades económicas de las Naciones, debido a que es un sector en desarrollo, que ha demostrado una fortaleza única, durante la crisis económica; un ejemplo de ello, se ve en la Unión Europea UE, donde las autoridades de este continente han reconocido la relevante aportación del deporte a su economía, específicamente, en la creación de puestos de trabajo y desarrollo económico local, y las consecuencias multiplicadoras, en otros sectores (Piñeiro, 2015).

En el 2012, el balance sobre la contribución del deporte al crecimiento económico en la UE fue el aumento del empleo en cuatro subsectores de la industria del deporte; el primero, el turismo activo, debido a la demanda interna en Suecia y Alemania, como también a los turistas internacionales, en Austria e Italia y el apoyo fundamental en el sector de la hostelería; el segundo, el fitness, prevalentemente en el Noroeste Europeo, Suecia y Holanda, gracias a la fuerte demanda comercial de deportes indoor; tercero, los medios de comunicación, aprovechando el efecto tractor del fútbol profesional y la comunicación en países, como el Reino Unido y finalmente, la educación deportiva, componente transversal de la economía en todos los países, especialmente, en Estonia, Dinamarca y Letonia (Piñeiro, 2015).
Otro dato relevante sobre la importancia de la innovación deportiva y sus orígenes, se observa en el 2014, tiempo en el que se generó el 2,12\% de ocupaciones en la UE, consiguiendo 4,46 millones de empleados; las consecuencias directas en el empleo ascendieron a 41.460 .888 de trabajadores y sumando los indirectos, lograron más de 71 millones de trabajadores (Piñeiro, 2015).

Como se observa, el área del deporte es una industria de trabajo intensivo; su crecimiento conduce a la producción de empleo adicional y, por lo tanto, necesitado de lograr un fenómeno innovador, creativo, novedoso del deporte, que favorezca la dimensión social, económica, cultural y tecnológica, alrededor del mundo. A continuación, un vistazo al origen de algunas de las nuevas disciplinas deportivas por el mundo.

El Stickball, se categoriza como actividad urbana y surgió como resultado de la práctica de los deportes en las calles, propios de deportes tradicionales de las comunidades de inmigrantes en Estados Unidos, en la década de 1960; se relaciona con el béisbol convencional y el streetball, una variante de baloncesto tradicional, una adaptación clara de ambos (García, 2017).

El Tchoukball, se define como un deporte de equipo, que se juega bajo techo. Fue desarrollado en los años 1970, por el biólogo suizo Hermann Brandt, quien creía que el objetivo de las actividades físicas humanas no era la competencia entre perdedor y ganador, sino aportar a la construcción de una sociedad cooperadora. Su objetivo era desarrollar un deporte de equipo, que no implicara contactos fuertes, como los que ocurren en otros deportes (Asociación Española de Tchoukball, 2017).

El Kickball fue inventado, aproximadamente, en 1917, por Nicholas C. Seuss, deporte que fue implementado en 1920, por profesores de educación física, en las escuelas primarias, para enseñar a los niños los fundamentos del béisbol (Urrutia, 2019).

El Buble Soccer tiene sus orígenes en Noruega, en el 2011, cuando fue transmitido un vídeo por el programa Golden Goal, en el que se mostraba esta manera tan distintiva de jugar al football, de una forma más segura y dinámica (Jones, 2014). 
Una fusión entre el Fútbol y el Billar es la combinación a la que se denomina Snookball, una modalidad que nació en Francia, gracias a dos jóvenes, Samuel y Aurélien y que, gracias al auge de las redes sociales, poco a poco, se ha hecho conocer en los países europeos (Domínguez, 2015).

El Bossaball es un deporte de equipo, que inició en España, creado por Filip Eyckmans, en el 2005; es un juego de pelota entre dos equipos, que combina elementos del voleibol, fútbol y gimnasia, con música, en un deporte (Bosaball, 2010; Lara, 2016).

El footgolf o fútgolf fue creado en Holanda, en el 2009, siendo uno de sus creadores Michael Jensen y es un deporte que se caracteriza por una fusión del fútbol y el golf, aunque se puede considerar que está más próximo al golf. A partir del 2012, se jugaron tres mundiales: el primero, en Hungría; en Argentina, para el 2016 y en Marruecos, en el 2018 (Mercedesya, 2020).

Y, finalmente, el Human Bowling. La mayoría de los investigadores plantean que no hay información sobre el año de origen de este deporte; sin embargo, se puede estimar el 2003, como el momento en el que este novedoso deporte funda y lanza Pistas de Bowling, con tecnología innovadora (Imply, 2018).

Como lo sostiene un experto, "...Ahora, los deportes tienen que ver con la habilidad individual y las estrategias de equipo, ya no van tanto a eliminar, al contrario, sino a realizar estrategias de ataque. Además, lo pueden practicar hombres y mujeres a la vez" (Beltrán, 2012). Por lo que se observa, un tránsito de la competencia al disfrute.

\section{Las nuevas disciplinas deportivas: cómo llegan a Colombia}

"El ingreso de Colombia al mundo global, con la reforma constitucional de 1991, no solo cambió los marcos de referencia en el campo económico, político, social y cultural en general, sino que afectó espacios específicos de la vida cotidiana", Thomas Beltrán.

De acuerdo con algunos investigadores, el deporte en Colombia, empieza con la regeneración, de 1886 a 1899 y el predominio conservador, desde 1900 hasta 1930; en esos tiempos, se hicieron muchos debates sobre la formación física y moral del pueblo y sobre el mejoramiento de la raza. En aquel periodo, se da la llegada de practicantes de distintas disciplinas deportivas al país, que tiene su cumbre con la reglamentación de la Ley 80 de 1925 (Congreso de Colombia, 1925), la cual, normaliza la educación física, los deportes y la creación de la Comisión Nacional de Educación Física, que sirvieron de base para la celebración de los Juegos Olímpicos de 1924 y 1926 y los Juegos Olímpicos Nacionales de 1928, celebrados en Santiago de Cali (Quitián, 2013).

Comenzaban, entonces, las prácticas tipo aficionado y la puesta en escena de los ideales olímpicos de Coubertin, con el concurso de la Asociación Colombiana de Fútbol (1924), el Instituto Nacional de Educación Física (INEF) (1936) y el Comité Olímpico Colombiano (1936) (Comité Olímpico Colombia, 2015).

De la misma manera, se iniciaba la masificación de los deportes, se fundaba la comunidad imaginada, término utilizado para designar nación, como, por ejemplo, los juegos nacionales, la vuelta a Colombia, el campeonato de fútbol, entre otros (Quitián, 2013); en ese momento, se renuncia a la idea de "aldea tradicional y se siembra el germen de la futura aldea global". Colombia, se afilia de manera inicial a la FIFA y al Comité Olímpico Internacional; de igual forma, juega en la Olimpiada de Berlín, en 1936, en el Torneo de Fútbol Sudamericano, en 1945 y en los Juegos Centroamericanos y del Caribe, de 1946.

A partir de allí, ocurrieron muchas cosas en el deporte nacional, fundamentales en los procesos de modernización y post-modernización de la sociedad colombiana, en los que la actividad física desempeñó un papel destacado, particularmente, en los campos del ciclismo, el fútbol y el patinaje (Sánchez, 2015).

Nunca, se habían presentado cambios tan radicales en el campo deportivo como cuando ingresó Colombia al mundo global. Con la reforma constitucional de 1991, no solo cambió los marcos de referencia en el campo económico, político, social y cultural, en general, sino que afectó espacios específicos de la vida cotidiana (Sánchez, 2015), entre ellos, se destacan las actividades deportivas, como Educación física, Ciencias aplicadas o Ciencias del deporte, influenciadas, de un día para otro, con una cantidad de nuevas disciplinas, importadas desde los campos 
de creación de nuevas rutinas físicas, surgidas de la interacción humana planetaria y de la tecnología (Bagnara \& Pacheco, 2011). Entre estas nuevas actividades, se pueden mencionar el Toukchball, que llegó al país en el 2009, a la ciudad de Cali. A partir de ese momento gustó y posteriormente, entró a Bogotá, donde ha causado furor en diferentes universidades y colegios. Es una mezcla entre balonmano, squash y voleibol y, como la gran mayoría de los nuevos deportes, el contacto físico es mínimo; de otro lado, el Kickball no es un deporte tan nuevo, pero en Colombia, tuvo su auge en la Costa Atlántica. Tiene similitud con el béisbol y un toque de fútbol. El lanzador arroja una bola pesada desde el centro hacia el receptor, este golpea el esférico con el pie y, luego, corre a la primera base (Beltrán, 2012; Shapiro et al. 2015).

En el país, hay un grupo de mujeres cartageneras que logró, con deportes tradicionales, como el fútbol, el baloncesto o el patinaje, dar lugar a nuevas disciplinas, que han causado sensación en jóvenes y adultos, que ven otra forma de realizar actividad física; igualmente, se puede agregar el Buble Soccer, la variante más loca del fútbol tradicional; el Snookball, como jugar billar con los pies; el Bossball, el voley para los amantes de las auténticas filigranas; el Foot golf, si se cuenta con un Remi Gaillard dentro, se podrá demostrar la calidad y la puntería; el 360 ball, otro innovador deporte de raqueta; el Human Bowling, deporte en el que se descarga toda la adrenalina (Pardo, 2016; Beltrán, 2012).

Al parecer son muy pocas las investigaciones y reflexiones que se han adelantado alrededor de un tema tan importante en Colombia. Incluso, como lo sostienen varios investigadores, como Olivera (2006), en Latinoamérica, la discusión sobre el deporte, como objeto de estudio, denota un insuficiente debate teórico, especialmente, con relación a los contextos sociales, políticos e ideológicos.

Por lo anterior, y como aportan distintos autores, las facultades de ciencias del deporte, disciplinas deportivas o ciencias aplicadas necesitan establecer la proyección social, es decir, aquellas acciones - actividades relacionadas con los campos anteriormente nombrados, que se están llevando a cabo, para la actualización en sus formas y contenidos pedagógicos, para que la formación de sus profesionales alcance los mejores niveles, de acuerdo con los requerimientos contemporáneos, establecidos, principalmente, por las coordenadas de las nuevas disciplinas, sin demeritar, por supuesto, la larga tradición y experiencia en los campos tradicionales de la formación deportiva (Zagalaz et al. 2001; López \& Gea, 2010).

\section{Prácticas de enseñanza y aprendizaje para los nuevos deportes}

"Ir...mucho más allá, intentando no solo aprender de forma diferente a desenvolverse en un juego, sino estimular la creatividad del alumnado como capacidad potencial en el ser humano para que nuestras sesiones tengan una mayor significatividad y utilidad", Daniel Moreno.

Resulta de vital importancia adelantar una exploración inicial de algunos de los deportes más sobresalientes en el mundo y que vienen teniendo un impacto notable, particularmente, entre los jóvenes colombianos. Esta visión inicial ofrece una clara perspectiva de tales disciplinas y sustenta la factibilidad y la pertinencia de la investigación, que dio lugar a este documento.

A continuación, se describirán los elementos más destacados de cada una de ellas.

El Tchoukball. Hermann Brandt creador de este deporte, consideraba que el objetivo de las actividades físicas humanas no es ser campeones, sino contribuir a la construcción de una sociedad armoniosa. Su objetivo era desarrollar un deporte de equipo, que no implicara heridas graves, como las que ocurren en otros deportes (Asociación Española de Tchoukball, 2017).

Está basado en el balonmano y tiene una gran riqueza táctica, ya que prima la colocación de los jugadores en el campo. La técnica, se reduce al pase, al lanzamiento y a la recepción. Es necesario un balón de balonmano o de tamaño similar y dos superficies de rebote. Algo peculiar en este deporte es que el balón no puede ser robado ni interceptado; la lógica, se encuentra en "dejar jugar", el equipo contrario puede recuperar el balón en el momento que este se encuentre en el aire y después de que haya golpeado la superficie del rebote. No existe un arco perteneciente a un equipo, lo que indica que cualquiera de los equipos puede utilizar cualquiera de las superficies de rebote (Noak \& Ruíz, 2014; Gomes, 2011). 
Existe también, el Tchoukball playa, que se creó en los años 90, en Brasil. A diferencia del Tchoukball estándar, este se juega con 5 jugadores por equipo en lugar de 7, aplicando las mismas normas del estándar, implica una gran rapidez y habilidad, en el que los jugadores se pueden expresar libremente mientras juegan; es de alto nivel, ya que se pueden anotar puntos cada 20 segundos. Es versátil porque se puede jugar en cualquier lugar (playa, césped, piscina, entre otros), además, está destinado para todas las edades, no cuenta con limitación de edad, altura, contextura o peso (Mejía, 2014).

Se ejecuta en una cancha de 29 metros de largo, por 17 metros de ancho, aproximadamente. En cada extremo hay un cuadro de rebote y un semicírculo, que mide de tres metros desde el arco, en todas las direcciones. Cada equipo puede anotar en ambos extremos en el campo y cuenta con siete jugadores por equipo. Para anotar punto, una de las bolas necesita ser lanzada por un jugador atacante, rebotar en el cuadro y picar en la cancha fuera del área, sin ser capturado por el equipo defensor. El contacto físico no se permite y los defensores no pueden intentar interceptar el pase del equipo atacante. Los jugadores pueden realizar tres pasos con el balón, mantenerlo durante un máximo de tres segundos en sus manos. Si un jugador no acierta al cuadro después de realizar un tiro, el equipo defensor obtiene un punto (García, 2013).

El Kickball, también llamado Kickingball o Fireball, se juega con dos equipos, el objetivo es anotar la mayor cantidad de carreras posibles y evitar que el otro equipo anote carreras. Gana el equipo que haya anotado más carreras en conformidad con el reglamento y bajo la jurisdicción de uno o más árbitros. El campo de juego es llano, irregular, con una sensación ovalada, compuesto por dos tipos de terrenos, primero, el terreno de foult y segundo terreno, bueno. Ambos espacios, están compuestos por distintas zonas, que integradas conforman todo el campo de juego del Kickball (García, 2013).

Este deporte, se clasifica en juegos y deportes colectivos, es competitivo y se practica, generalmente, en Norteamérica; sin embargo, es el juego favorito de la juventud en Corea del Sur, donde se conoce como balyagu (pie-béisbol), también es uno de los deportes que se practica, regularmente, en las escuelas primarias de varios continentes (García, 2013).
Un juego de Kickball está integrado por dos equipos de 9 jugadores, el fin es anotar más carreras que su oponente, recorriendo las 3 bases y cruzando el plato del "home", tantas veces como sea posible. El balón oficial es rojo, con un logotipo de WAKA y se infla a 1,5 libras por pulgada cuadrada. El equipo visitante inicia pateando, mientras el equipo local comienza en el campo. El primer pateador sube al plato para iniciar el juego. Los jugadores, se alinean en las siguientes posiciones: 1 . Lanzador (en el caucho de picheo), 2. Cátcher (detrás de la placa del hogar), 3. Primera base (a pocos metros de la primera base), 4. Segunda base (a pocos metros de la 2 a base hacia la 1ra), 5. Tercera Base (a pocos metros de la 3 a base), 6 . Parada de emergencia (entre 2a y 3 a base), 7. Jardín izquierdo (detrás de la segunda y tercera base), 8. Centrocampista (detrás de la 2a base) y 9 . Jardín derecho (detrás de 1ra y 2 da base) (Pyle et al. 1943).

A medida que creció el interés por este deporte, se formaron organizaciones, como la Asociación Mundial de Kickball de Adultos y miles de adultos participaron en el juego; fundada en 1998. Cuatro jóvenes, se establecieron en la idea de lanzar una liga deportiva, que mezclara atletismo e interacción social. Ellos eligieron el Kickball, debido a que requiere poca habilidad atlética y era poco probable que alguien se lastimara (Pyle et al. 1943).

El Buble Soccer. Este deporte es reconocido por su novedosa forma, dinámica y divertida de jugar fútbol; se convirtió en una gran innovación en Europa y sigue creciendo rápidamente, por todo el continente europeo. Algunos datos interesantes, se encuentran en España, en donde ya se han realizado torneos interuniversitarios, además de la investigación en neurociencias, que este deporte ha suscitado (Bubble Soccer MX, 2016; Halani et al. 2016).

Todos los jugadores necesitan ubicarse dentro de pelotas o burbujas, conocidas como Bumperz e intentan hacer gol en el arco opuesto; al mismo tiempo, sus contrincantes intentan derribarlos chocando con ellos, por supuesto, es una forma segura, dado que los jugadores no sufren golpes físicos, porque están protegidos por el Bumperz. Gana el equipo que anote la mayor cantidad de goles al opositor (Bubble Soccer MX, 2016).

El Snookball. Fusión entre el Fútbol y el Billar, también se le conoce como Fútbillar, tiene como 
finalidad poner a prueba la técnica y habilidad con los pies, que hacen las veces de "tacos", mientras que las bolas son sustituidas por balones, todo esto en una mesa de billar gigante. En este juego, a diferencia del balompié, no es necesario estar uniformado y tampoco usar zapatos especiales, basta con la clase que poseas en tus extremidades inferiores, para introducir los balones en los seis hoyos disponibles, con las mismas reglas que el billar (Domínguez, 2015).

El nombre proviene de la palabra Snooker, que es el juego de billar, que se practica con tacos más largos que los habituales, en una mesa de billar común, con una bola blanca, que debe golpear a 15 bolas rojas y a seis de otros colores (Domínguez, 2015).

Este deporte, se clasifica en deportes de adversarios, requiere de habilidad y de técnica. Aquí, los tacos se reemplazan por los pies y las bolas por pelotas, parecidas a las de fútbol sala; la pelota de color blanca es más pesada que las otras, dándole un toque más atrayente. El objetivo es similar al del billar, insertar las bolas en alguno de los seis hoyos de la mesa. Las dimensiones de la pista se acomodan para la organización de torneos de dos contra dos; durante el juego, se utilizan distintas técnicas de golpeo, para que las pelotas tengan efecto y puedan entrar en las canastillas (Domínguez, 2015).

El Bossaball. Se trata de un deporte de equipo que combina elementos del voleibol, fútbol, gimnasia y música, se juega en una cancha inflable con un trampolín, en cada lado de la red. Los trampolines permiten a los jugadores rebotar, lo suficientemente alto, como para rematar la pelota sobre la red y marcar puntos directos. Son distintos los países en los que se ha introducido el Bossaball, como Brasil, Argentina, México, Turquía, Países Bajos, España, Alemania, Francia, Suiza, Portugal, Grecia, Eslovenia, Hungría, República Checa, Rumania, Israel, Egipto, Arabia Saudita, Qatar, Kuwait, Chile, Ecuador, Venezuela, Paraguay y Colombia (Bosaball, 2010).

El objetivo es lograr colocar la pelota en el campo del equipo contrario. Cada equipo tiene un máximo de ocho toques dentro de su campo. El jugador, en la cama elástica, tiene que saltar hacia la posición correcta para lograr un "smash".

El balón puede tocar cualquier parte del cuerpo, pero solo una vez en la mano y dos veces consecutivas en el pie o la cabeza.
Toque de vóleibol: tocar la pelota con las manos o los antebrazos.

- Toque de fútbol: Tocar la pelota con otra parte del cuerpo que no sean los brazos; doble toque permitido (cuenta como un solo toque).

Cada toque, se hace cuando el balón toca el campo del oponente. Si el balón toca la cama elástica contraria, hace tres puntos. Lo primordial en el Bossaball es la coordinación en los movimientos y, a la vez, acostumbrarse a las caídas, pues los jugadores se pasan la mayor parte del juego en el aire que en pie; sin embargo, el juego es bastante seguro (Bosaball, 2010).

El Footgolf. Se caracteriza por una fusión del fútbol y el golf, aunque se puede considerar que está más próximo al golf, en que los jugadores golpean un balón de fútbol, con el objetivo de introducirlo en los hoyos, en el menor número de golpes posibles (BBC, 2017).

Este deporte guarda similitud con las del golf, destacándose en que, para este caso, se utiliza un balón de fútbol, en lugar de las bolas típicas del golf y en lugar de los palos del golf, se usan los pies. Los jugadores deben introducir el balón en los hoyos, que son de un tamaño acorde al del balón (Launicafm, 2019).

El juego consiste en transitar un círculo de 9 o 18 hoyos. El jugador que complete el círculo con el menor número de toques con el pie es el ganador. Desde el primer golpe con el balón, se busca alcanzar el hoyo, el jugador se enfrenta con variados retos, por ejemplo, árboles, lagunas, desniveles y otros elementos que forman parte del espacio de juego. Es, por tanto, la relación entre técnica, exactitud y fuerza utilizada; por lo tanto, las habilidades de los jugadores desempeñan un rol fundamental en el desarrollo del juego (Launicafm, 2019).

El Human Bowling. Este deporte plantea la existencia de libertad y de flexibilidad creativa para hacer uso del Human Bowling, como un espacio para la presentación de la especificidad, de la recursividad y de la diversidad de las formas y variantes, señaladas anteriormente. Aparte de la diversidad de formas de ejercicio, técnicas y jugadas, su aplicabilidad es un aprendizaje permanente, progresivo e independiente de los gestos técnicos. Y, en estos procesos virtuales o presenciales, se acumula, tanto conocimiento 
como experiencia, desde la diversidad de variantes implementadas que, mediante el proceso de práctica vivencial, facilitan los aprendizajes de multitud de juegos, ejercicios y técnicas deportivas (BaenaExtremera et al. 2013).

\section{RESULTADOS}

La revisión documental adelantada en el proceso de exploración de las nuevas disciplinas deportivas estableció, de un lado, hasta qué punto se han extendido estas prácticas en la vida cotidiana de la población colombiana, particularmente, entre los jóvenes. Aunque los deportes tradicionales siguen desempeñando un papel destacado, la incursión de otras actividades de cultura física está permeando la vida del país con mucha fuerza. De otro lado, aún es muy poco lo que se ha investigado acerca de tales acontecimientos, teniendo en cuenta su elevado influjo en la cultura global experimentada, en algunas ciudades principales, como Bogotá, Medellín, Barranquilla, entre otras. En el campo institucional, raramente, se habla al respecto, en términos académicos y en la educación, básica, secundaria e, incluso, universitaria, a pesar que se promociona la práctica de las nuevas disciplinas, se invierte poco en hacer realidad su abordaje investigativo.

Lo anterior, también indica la ausencia de innovación pedagógica en estas áreas y la necesidad de lograrlo, dado que los estudiantes y practicantes de las nuevas disciplinas requieren de herramientas teóricas y vivenciales, que les permita abordarlos desde visiones nuevas, ajustadas al espíritu deportivo, impulsado por las nuevas tendencias del deporte mundial y colombiano, tal como lo afirma Peire \& Estrada (2015).

Igualmente, es necesario anotar la nueva imagen corporal auspiciada por las nuevas prácticas deportivas, como la delgadez, la fortaleza física, el autocuidado sanitario, el control dietario, entre otras.

Asimismo, estamos hablando del concepto de postdeportes, seguramente relacionado con el posthumanismo (categoría que hace referencia a las nuevas formas de organización social, producto de los desarrollos tecnológicos y científicos contemporáneos), definido como prácticas circulares, flexibles, fugaces, en donde el espectáculo y los dispositivos tecnológicos son su condición de existencia; cabe anotar, la independencia institucional como atributo notable de su ejercicio. El descenso en la práctica de los deportes modernos tradicionales tiene consecuencias inmediatas y deja el lugar a las nuevas prácticas lúdico-deportivas, que se pueden realizar en espacios colectivos no convencionales, deportivizando todo el país en sus ciudades.

\section{DISCUSIÓN}

No cabe la menor duda que en Colombia, particularmente en su capital y principales ciudades, se necesita la reflexión profunda acerca del impacto de los vertiginosos cambios que se han venido presentando en los últimos treinta años. Desde la economía, la política, el contexto social y la cultura, se han presenciado transformaciones imposibles de creer no hace mucho tiempo. Del conocimiento que se obtenga alrededor de tales cambios depende no solo de la ubicación del país en los acontecimientos del futuro próximo, sino también, la propia supervivencia de las instituciones encargadas de tramitar los ajustes pedagógicos visionales y misionales necesarios, cuando se presentan estas situaciones.

Dado lo anterior, los ajustes implican la introducción de nuevas formas educativas orientadas a entender, entre otras, las formas y los contenidos de las nuevas disciplinas deportivas, entendidas, ahora, como actividades entrelazadas, con muchas áreas del conocimiento, que las potencian en el cumplimiento de sus propósitos transformadores.

Otro elemento crucial en esta discusión, se relaciona con la proyección social de estas nuevas prácticas, pues involucran el entorno familiar y social, como elementos clave del disfrute del deporte, en las más recientes visiones culturales.

De la misma manera, se necesita considerar el tema de los espacios de práctica de las nuevas disciplinas deportivas, ya que, normalmente, las áreas de juego, se caracterizaban por ser territorios de competencia, circunstancia casi completamente ausente, en estas novedosas actividades.

Ciertamente, Colombia se ha destacado en muchas disciplinas deportivas en los últimos años, como el fútbol, el ciclismo, el patinaje, la natación, el boxeo, las pesas, no únicamente en lo local y lo regional, sino también a nivel olímpico, pero ¿De qué manera se incorpora el país en estas nuevas disciplinas deportivas? ¿Cómo las ingresa en la legislación y 
en las normas del deporte colombiano? ¿En qué ajustes educativos, pedagógicos, epistemológicos se comprometen las instituciones formadoras de estudiantes, docentes, entrenadores para alcanzar los exigentes niveles presentes en estas nuevas prácticas?

Se trata de muchas indagaciones que necesitan de respuestas para que el deporte colombiano continúe sus exitosos recorridos en el tiempo y el espacio que, hoy, experimenta el país.

\section{CONCLUSIONES}

Al final de este ejercicio investigativo, se presentan las inferencias más destacadas, producto del análisis y procesamiento de la información obtenida y que se quiere compartir con el lector, particularmente, con las entidades académicas formadores de los profesionales, protagonistas principales de las ciencias del deporte.

Lo primero que se destaca es el hecho de que, a nivel internacional, se ha producidomucha documentación, producto de variadas investigaciones adelantadas por expertos en las nuevas disciplinas deportivas y de diferentes países, especialmente, europeos.

En segundo lugar, la constatación de la cantidad de nuevas actividades deportivas surgidas en el mundo, particularmente en Europa; algunas de ellas, surgidas de las combinaciones de actividades físicas existentes previamente y otras completamente nuevas.

En tercer lugar, el cambio profundo de la visión en las actividades deportivas, más enfocadas en el disfrute y en el compartir que en la competencia.

En cuarto lugar, se destaca la forma completamente improvisada e inesperada de llegar las nuevas disciplinas deportivas a suelo colombiano. Hasta donde se sabe, muy pocas instituciones le han prestado la atención necesaria, a este fenómeno tan importante.

En quinto lugar, la poca preparación de las instituciones relacionadas con el deporte para adecuar estas prácticas a las condiciones y a las necesidades colombianas.

En sexto lugar y quizá lo más importante, la oportunidad que se abre a la sociedad colombiana y sus instituciones educativas, para incorporar las nuevas prácticas en la vida cotidiana, principalmente, en su ciudad capital y ciudades principales.

Finalmente, a partir de los resultados aquí presentados, se abren enormes posibilidades para adelantar investigaciones aún más amplias y profundas alrededor de las Nuevas Disciplinas Deportivas que le ofrecen a la sociedad colombiana, a las facultades de cultura física y deportes, una amplia gama de temas y puntos de interés, para promover la cultura de la investigación y el cambio para lograr la actualización cultural requerida por el país, en estos tiempos tan complejos.

\section{REFERENCIAS}

1. ASOCIACIÓN ESPAÑOLA DE TCHOUKBALL. 2017. La Historia del Tchoukball. AETB. Disponible desde Internet en: http://tchoukballspain.com/ historia-del-tchoukball

2. BAENA-EXTREMERA, A.; SERRANO, J.; FERNÁNDEZ, R.; FUENTESAL, J. 2013. Adaptación de nuevos deportes de aventura a la educación física escolar: las vías ferratas. Apunts. Educación Física y Deportes. 114:36-44.

3. BAGNARA, C.; PACHECO, I. 2011. El desarrollo tecnológico en las actividades físicas y el deporte. Efdeportes. 159:1-4.

4. BBC. 2017. Qué es el footgolf, el curioso deporte mezcla del fútbol y el golf que es un fenómeno global. BBC News Mundo.

5. BELTRÁN, T. 2012. El país se llena de los deportes más novedosos. Publimetro. 1-30.

6. BOSABALL. 2010. Un deporte radicalmente diferente. Disponible desde Internet en: https://www. bossaballsports.com/es/bossaball-2/que-esbossaball/

7. BUBBLE SOCCER MX. 2016. ¿Qué es Bubble Soccer? Disponible desde Internet en: https://www. bubblesoccer.mx/que-es-bubble-soccer

8. CONGRESO DE COLOMBIA. 1925. Ley 80 de 1925, sobre educación fisica, plazas de deportes y precio de becas nacionales. Disponible desde Internet en: http://www.suin-juriscol.gov.co/ viewDocument.asp?id=1625996 
9. DOMíNGUEZ, G. 2015. Snookball: al billar también se juega con los pies. Libertad Digital. Disponible desde Internet en: https://www.libertaddigital. com/deportes/mas-deporte/2015-01-22/elsnookball-el-ultimo-deporte-que-causa-furoren-medio-mundo-1276538830/

10. FUSTER, J.; FUNOLLET.; F.; GÓMEZ, J. 2008. Características Elementales de los Nuevos Deportes en el Medio Natural. Ágora para al EF y el Deporte. 7-8:35-47.

11. GARCÍA, A. 2013. Deportes Alternativos. Disponible desde Internet en: https://es.slideshare.net/alberto.garciahuerta/ deportes-alternativos-13-14

12. GARCÍA, S. 2017. Estudio comparativo de la práctica de actividades físico-deportivas en los espacios urbanos entre universitarios italianos y españoles. Tesis Doctoral. Universidad Pablo de Olavide (Sevilla). Disponible desde Internet en: https://rio.upo.es/xmlui/handle/10433/4742

13. GARCÍA, S.; FERNÁNDEZ-TRUAN, J. 2018. Génesis de los Deportes Urbanos. Athenea Digital. 18(1):349-364

14. GOMES, R. 2011. Tchoukball, un nuevo deporte en las arenas. El Globo. 7-15.

15. HALANI, S.; RILEY, J.; PRADILLA, G.; AHMAD, F. 2016. How safe is Bubble Soccer. Journal of Clinical Neuroscience. 34:227-229. https://doi.org/10.1016/j.jocn.2016.08.007

16. HERNÁNDEZ, R.;FERNÁNDEZ, C.; BAPTISTA, P. 2014. Metodología de la Investigación. McGRAW-HILL (México). 6a edición. 600p.

17. IMPLY. 2018. La história del Bowling. Disponible desde Internet en: https://www.imply.com/ es/entretenimiento/la-historia-del-bowlingmilenario/\#: :text=Siglo\%20XIX\%3A\%20La\%20 versi\%C3\%B3n\%20de,estandarizar\%20y\%20 mejorar\%20las\%20normas

18. JONES, S. 2014. Bubblesoccer. Disponible desde Internet en: https://www.bubblesoccer.mx/ information

19. LARA, B. 2016. Plan de negocios para la creación de un club de bossaball. Tesis de pregrado. Universidad de las Americas (Quito, Ecuador). Disponible desde Internet en: http://dspace.udla.edu.ec/handle/33000/5931
20. LAUNICAFM. 2019. Footgolf: El Nuevo Deporte que también arrasa en Talavera. Launicafm. Disponible desde Internet en: https://www. launicafm.com/footgolf-el-nuevo-deporte-quetambien-arrasa-en-talavera/

21. LÓPEZ, V.; GEA, J. 2010. Innovación, Discurso y Racionalidad en Educación Física. Revisión y Prospectiva. Revista Internacional de Medicina y Ciencias de la Actividad Física y del Deporte. 10(38):245-270.

22. MEJÍA, D. 2014. Caracterizacion Del Tchoukball. Calameo. Disponible desde Internet en: https://en.calameo.com/ $\mathrm{read} / 00590706348131 \mathrm{fe} 8955 \mathrm{~d}$

23. MERCEDESYA. 2020. Qué es el Footgolf que este domingo se juega en Navarro. Disponible desde Internet en: https://mercedesya.com/noticias/ que-es-el-footgolf-que-este-domingo-se-juegaen-navarro-zuxtfqb

24. NOAK, P.; RUÍZ, D. 2014. Introducción al Tchoukball en el Ámbito Escolar como Medio para Mejorar y Fortalecer las Habilidades Básicas Motoras. Universidad Libre de Colombia (Bogotá).

25. OLIVERA, J. 2006. El sistema deportivo. Amenazas y oportunidades. Apunts Educación Física y Deportes. 85:3-6.

26. PARDO, Y. 2016. Escuela de Kickball, una Apuesta por el Desarrollo de las Niñas y Jóvenes Clemencieras desde el Deporte. Universidad de Cartagena (Cartagena).

27. PEIRE, T.; ESTRADA, J. 2015. Innovación en la Educación Física y en el Deporte Escolar: Métodos de Enseñanza, Deportes y Materiales Alternativos. e-balonmano.com Revista de Ciencias del Deporte. 11:223-224.

28. PIÑEIRO, S. 2015. Contribución de la innovación deportiva al crecimiento económico europeo. SporTK-Revista EuroAmericana De Ciencias Del Deporte. 4(2):25-32. https://doi.org/10.6018/242911

29. PYLE, C.; ERNIE, A.; KELLY, L.; ORR, O. 1943. Here Is Your War. Story of G.I. Joe (Nueva York).

30. QUITIÁN, D. 2013. Deporte y modernidad: caso Colombia del deporte en sociedad a la deportivización de la sociedad. Revista Colombiana de Sociología. 36(1):19-42. 
31. RADOJEVIĆ, J.; GRBOVIĆ, M.; JEVTIĆ, B. 2019. Academic Study Programs and Education for the Profession of Sports Coach Occupation. Review Paper. 73(2):90-106.

32. SÁNCHEZ, R. 2015. Modernización, Actividad Física y Reproducción Social. Una Antropología de las Culturas Deportivas Contemporáneas. Tesis doctoral. Universitat Ramon Llull (España).

33. SHAPIRO, D.; MASTRO, J.; BALMER, S. 2015. Bip Kickball: un deporte adaptado para personas con pérdida de visión de todas las edades. Palestra. 29(1):37-42.
34. URRUTIA, M. 2019. Esta es la historia. Disponible desde Internet en: https://estaeslahistoria. com/c-deportes/historia-del-kikimbol/

35. ZAGALAZ, M.; MORENO, R.; CACHÓN, J. 2001. Nuevas Tendencias en la Educación Física. Contextos Educativos. 4:263-294. 